\title{
Infections in Diabetes and Study of Outcome in Relation to Mortality and Morbidity
}

\author{
Dr.TVD Sasisekhar ${ }^{1}$, Dr.Aravindsriramc ${ }^{2}$, Dr.Shefali A ${ }^{3}$, Dr. Chetan Somu ${ }^{4}$, \\ Dr.Hari Krishna $\mathrm{B}^{5}$. \\ ${ }^{\text {I}}$ (Professor \& HOD, Department Of General Medicine, Dr. Pinnamaneni Siddhartha Institute Of Medical \\ Sciences \& Research Foundation, Chinoutpalli, Andhra Pradesh, India) \\ ${ }^{2}$ (Assistant Professor, Department Of General Medicine, Dr. Pinnamaneni Siddhartha Institute Of Medical \\ Sciences \& Research Foundation, Chinoutpalli, Andhra Pradesh, India) \\ ${ }^{3,4,5}$ (PG Student, Department Of General Medicine, Dr. Pinnamaneni Siddhartha Institute Of Medical Sciences \\ \&Research Foundation,Chinoutpalli, Andhra Pradesh, India)
}

\begin{abstract}
:
Aims: To study the cause of infections in diabetic individuals and outcome of patients in relation to mortality and morbidity.

Materials And Methods: The study group comprised of 100 diabetic patients who were admitted in Dr. PSIMS $\& R F$ with history of fever between July 2014 and July 2016. Age, sex, duration of diabetes mellitus, presence of fever, associated complaints and treatment history were documented. Outcome of these was assessed based on duration of stay in hospital and other parameters like disability and mortality.

Results: Soft tissue infections were most common infections (38\%) followed by respiratory tract infections (28\%) and genitourinary infections $(20 \%)$ in our study. Staphylococci $(25 \%)$ constituted the major cause of infections followed by E. coli (13\%), Klebsiella (12\%), and Pseudomonas (12\%). There was no correlation between prevalence of various infections and glycaemic levels $(p>0.5)$. However, their duration of stay and mortalitywas correlated with levels of glycated hemogolobin and it was found to be significantly related $(p<0.0001)$.
\end{abstract}

Keywords: Diabetes mellitus, Glycated hemoglobin, Infections, Outcome, Polymorphonuclear cells

\section{Introduction}

Globally, the number of people with Type 2 diabetes or diabetes mellitus is projected to grow to $366-$ 440 million by 2030, with three quarters of the increase in low- to middle-income countries. [1]India leads the world with largest number of diabetic subjects earning the dubious distinction of being termed the "diabetes capital of the world".

Before the discovery of insulin and antibiotics, infections contributed substantially to diabetesassociated morbidity and death. It has been estimated that infections killed 1 in 5 diabetic patients in the 1920s compared to $<1$ in 20 in the late 1960 's. Despite recent advances in the management of both diabetes and infectious diseases, diabetic patients remain at increased risk of infection. [2]

Some of the infections in diabetics occur with increased frequency and severity while others areexclusively associated with diabetes. Recent findings support a causal relationship between high blood glucose and infection as evidenced by a decrease in morbidity with improved glycemic control $[3,4]$ and worsening of diabetes during infection. Several factors contribute to increased predilection for infection which includes, most importantly, an altered immunity. The innate immune system is predominantly affected manifesting as dysfunction of polymorphonuclear cells, [5] as well as monocytes and macrophages.

Several polymorphonuclear (PMN) defects occur in diabetic subjects, including impaired migration, phagocytosis, intracellular killing, and chemotaxis which may be due to decreased PMN membrane fluidity. Generalized immunologic defects such as these raise the suspicion that diabetic patients may be at an overall increased risk for infection. [6]

Although several mechanisms have been postulated, it is currently believed that advanced glycation end products (AGEs) have a role in immune dysfunction.Hyperglycemia or presence of AGEs correlate with low level, persistentactivation of PMNL's [5] characterized by spontaneous oxidative burst and releaseof enzymes like myeloperoxidase, elastase and neutrophill granule components.The end result is an exhausted PMNL incapable of adequate response to achallenge by an infectious pathogen. [7] Tolerance as a result of spontaneoushyper excitement, has also been reported in peripheral blood mononuclear cells indiabetic patients.

Superoxide production is reduced in parallel with increasing glycemicexposure, $[8,9,10]$ especially at ambient glucose concentrations $>12 \mathrm{mmol} / \mathrm{L}$. [5] Thereduced superoxide production may reflect an increase in polyol pathway activityas a consequence of raised intracellular glucose. [11,12] As an electron donor,NADPH is

DOI: 10.9790/0853-1601104144 $\quad$ www.iosrjournals.org $\quad 41 \mid$ Page


a necessary part of the polyol pathway and its consequently increasedutilization may be at the expense of reduced levels of superoxide production.In tissues that do not need insulin for glucose transport, the hyperglycemicenvironment increases intracellular glucose levels, which are then metabolized, using NADPH as a cofactor. The decrease in the levels of NADPH prevents theregeneration of molecules that play a key role in antioxidant mechanisms of thecell, thereby increasing the susceptibility to oxidative stress. [13]

\section{Materials And Methods}

The study was conducted in Dr. Pinnamaneni Siddhartha Institute of Medical Sciences \& Research Foundation, Chinnoutapalli. Patient or patient's attendants consent was taken and ethical clearance was obtained from institute's ethical committee. 100 patients who were admitted in the hospital with febrile illness during the period July 2014 to July 2016 between the age groups of 18 and 79 of both sexes who were selected through simple random sampling were included in the study.Details pertaining to age, sex, duration of DM, presence and duration of fever, symptoms of respiratory, genitourinary, soft tissue disease, history of any trauma were documented along with treatment history.

Fasting blood sugar,Post prandial blood sugar,HbA1c,Complete blood count, ESR, blood culture and sensitivity, Skin-KOHpreparation in case of suspected fungal infections, wound swab for culture and sensitivity in cases of cellulitis, urine routine and culture and sensitivity obtained through early morning mid-stream urine sample were sent, Chest X-ray PA view, X- ray PNS, throat swab, endotracheal tube secretions and sputum for AFB and culture sensitivity were done in respiratory infections, spinal fluid was drawn by lumbar puncture and analysis was done for biochemical, microbiological parameters in CNS infections.

The statistical analysis is done using the SPSS version 18, Chi-square test has been used to find the significant association of glycated haemoglobin with parameters like duration of stay and mortality among study population.A p-value of $<0.05$ was considered as statistically significant.

\section{Results}

Mean age of study population was $48.0 \pm 10.0$ years. $53 \%$ of population were males and $47 \%$ were females in whom relation between long term diabetes control andoutcome were studied.

Out of 100 patients, 38\% were having skin and soft tissue infection (SSTI), 28\% were suffering from respiratory tract infections, $20 \%$ had genitourinary infection, $7 \%$ had gastrointestinal infections, $4 \%$ had blood related infections and $3 \%$ had CNS infections. Staphylococci constituted the major cause of infections i.e. $25 \%$ of total infections of which 12 cases were infected by coagulase negative staphylococcus (CONS) followed by E.coli, Klebsiella, Pseudomonas (Fig 1).

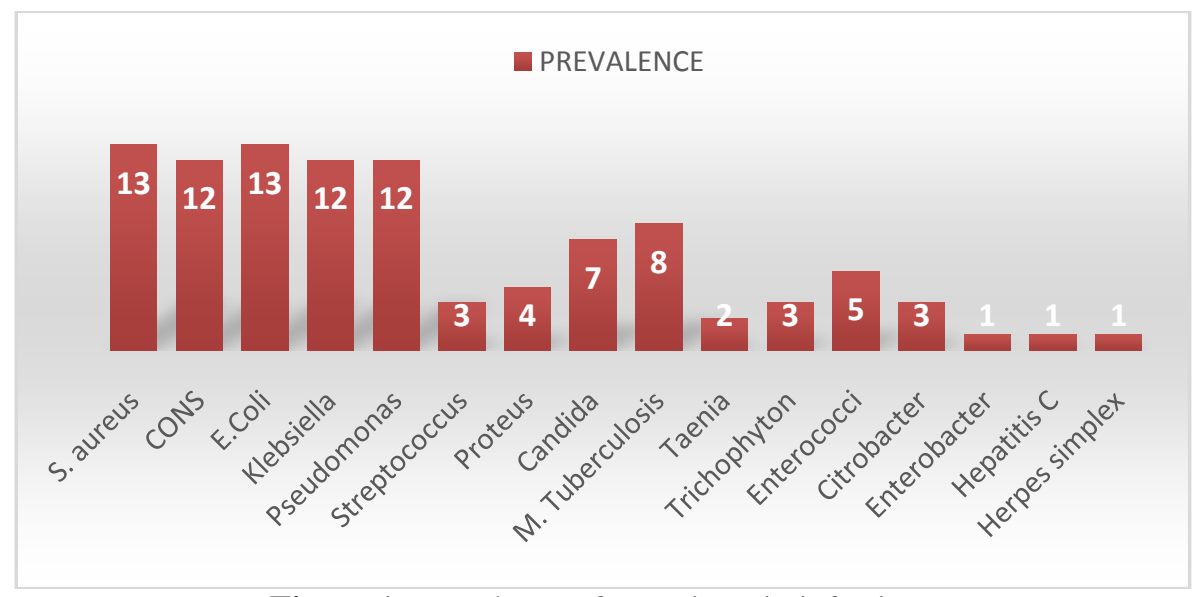

Figure 1: prevalence of organisms in infections

Diabetic foot is the major soft tissue infection in this study constituting $36 \%$ of the soft tissue infections followed by cellulitis $20 \%$, fungal infections $13 \%$, carbuncle $10 \%$. Rest of the infections i.e., otitis externa, gluteal abscess and perianal abscess constitute only a minor part of population. There were total 28 cases who suffered from respiratory tract infections, of whichpulmonary tuberculosis, pneumonia and ARDS constituted the majority.Twenty cases constituted genitourinary infections in this study. Thirteen patients were suffering from lower UTI infections, three patients in this group were suffering from pyelonephritis, three patients with vulvovaginitis and one patient had perinephric abscess.E.coli was the major responsible etiological agent for urinary tract infections which constituted $41 \%$, Klebsiella pneumoniae and coagulase negative staphylococcus constituted $18 \%$ and $17 \%$ respectively for UTI. 
$5 \%$ of the study population was disabled as they had undergone amputation and disarticulation for their underlying infections which was done as a life saving measure. Mortality was among $12 \%$ of the study population, of which UTI and respiratory tract infections constituted $33.33 \%$ each followed by skin \& soft tissue infection; CNS infection; blood infection contributing 16.66\%; 8.33\% and 8.33\% respectively (Table 1).E. coli was the responsible organism in causing $25 \%$ of the deaths, followed by Klebsiella species. Other organisms were Staphylococci, Citrobacter, Mycobacterium, HSV-1, Enterobacter and Pseudomonas.

Table 1: Table showing prevalence of infections and their outcome

\begin{tabular}{|l|c|c|c|c|c|}
\hline \multicolumn{1}{|c|}{ Infections } & \% of cases & Male & Female & $\begin{array}{c}\text { Mean DOS } \\
\text { (days) }\end{array}$ & $\begin{array}{c}\text { No. of cases } \\
\text { expired }\end{array}$ \\
\hline Soft tissue infections & 38 & 23 & 15 & 23.26 & 2 \\
\hline Respiratory tract infections & 28 & 15 & 13 & 17.18 & 3 \\
\hline Genitourinary infections & 20 & 8 & 12 & 18.45 & 4 \\
\hline GIT infections & 7 & 2 & 5 & 13.29 & 0 \\
\hline Blood infections & 4 & 4 & 0 & 14 & 2 \\
\hline CNS infections & 3 & 1 & 2 & 29 & 1 \\
\hline Total & 100 & 53 & 47 & $19.7 \pm 8.36$ & 12 \\
\hline
\end{tabular}

Mean duration of stay in this study among entire study population was $19.7 \pm 8.36$. Among the infections in our study CNS and soft tissue infections had 29 and 23.26 days as mean duration respectively followed by genitourinary and respiratory tract infections.

Mean FBS \& PPBS of the patients in the mortality group were 267.67 and 289.08 respectively and their SD were 127.58 for FBS and 98.85 for PPBS. There is no significant relation between FBS \& PPBS of the patients with the mortality.

Correlation between HbA1c and mortality was assessed. The HbA1c mean among people who had expired was 10.47 with SD of 0.77 and that of those who hadsurvived was 8.49 with SD of 0.82.Significant correlation was found between HbA1c and mortality (Table 2).

Table 2: Mean HbA1c and its relation with mortality

\begin{tabular}{|c|c|c|c|c|c|c|c|}
\hline Variable & Mortality & $\mathbf{N}$ & Min & Max & Mean & SD & P-value \\
\hline \multirow{3}{*}{ HbA1c } & Death & 12 & 9.4 & 12.0 & 10.47 & 0.77 & \multirow{2}{*}{0.0001} \\
\cline { 2 - 8 } & Survived & 88 & 6.9 & 12.2 & 8.49 & 0.82 & \\
\cline { 2 - 8 } & Total & 100 & 6.9 & 12.2 & 8.73 & 1.03 & \\
\hline
\end{tabular}

Mean duration of stay among survived group was $20.66 \pm 8.44$ days and among mortality group was $16.33 \pm$ of 7.20 days. Duration of stay of entire population irrespective of mortality is highly significantly related with $\mathrm{HbA1c}$ with $\mathrm{p}<0.0001$ and $\mathrm{r}$ value of 0.52 (Fig 2).

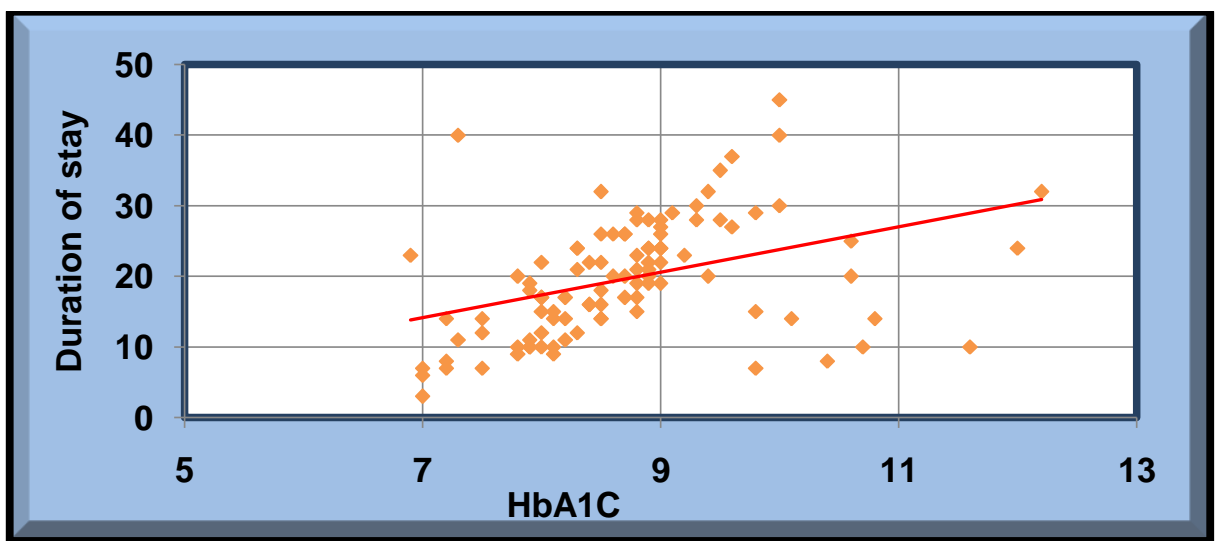

Figure 2: correlation between HbA1c and duration of stay among study population

\section{Discussion}

Diabetes mellitus (DM) is a clinical syndrome associated with deficiency of insulin secretion or action. It is the most common chronic metabolic disorder which is associated with high mortality and morbidity. Increased morbidity and mortality in diabetes is often caused by chronic complications, like coronary artery disease, peripheral vascular disease (diabetic foot), renal failure and retinopathy. In contrast to this, in India infection continues to be the most common cause of mortality. [7] 
Soft tissue infections contributed $38 \%$ of the total patients in the study, followed by respiratory infections (28\%) and genitourinary infections (20\%) which is similar to the other study conducted by Shariq R. Masoodi and others. [14] Contrary to the belief that urinary tract infections are most common in diabetics, in our study it was the third most common infection after soft tissue and respiratory tract infections.Unawareness of the importance of skin and foot care could be a reason for the higher prevalence of diabetic foot infections. This could also be attributed to the increased prevalence of diabetic foot at an average age of 50 years by which time they would have had developed neuropathy predisposing them to foot infections.

The HbA1c mean among people who expired was 10.47 with SD of 0.77 and the mean of people who have survived was 8.49 with SD of 0.82 correlation between $\mathrm{HbA1c}$ and mortality was statistically highly significant with a $\mathrm{p}$ value of $<0.0001$.A study done in Japan in 3,710 patients by Nakanishi.S and others [15] suggest that individuals with $\mathrm{HbA1c}$ levels of $6 \%$ or more might have increased mortality risk. But in this study the minimum value of $\mathrm{HbAlc}$ among the expired population was $9.4 \%$ and cut-off for mortality was taken as 10.15. This difference could be because majority of the population in this study were critically ill.

Study done by Halkos.M and others[16] shows that elevated HbA1c $\geq 8.6 \%$ caused a four-fold increase in mortality in post-operative patients of coronary artery bypass grafting.Study done by Ivan Gornik and others [17]shows relationship betweenhospital mortality and hospital length of stay. Admission HbAlc was significantly lower in surviving patients than in non- survivors $(\mathrm{P}<0.001)$ and there was a significant correlation between admission $\mathrm{HbAlc}$ and hospital length of stay of surviving patients $(\mathrm{r}=0.29$; $\mathrm{P}<0.001)$ in the abovementioned study. A study done by Frisch and others [4] shows patients with T2DM had significantly higher LOS in the hospital and significantly higher ICU LOS compared to non-diabetes subjects (8.8-10.6 days vs. 7-10.8 days and 2.3-6.2 days vs. 1.8-6.5 days respectively). The above two studies show similar results with the present study in terms of relation between $\mathrm{HbAlc}$ and duration of stay and between $\mathrm{HbA} 1 \mathrm{c}$ and mortality.

\section{Conclusion}

Diabetic patients have a high prevalence of infections. Infections in diabetes are more in the 4th and 5 th decade. There was no difference regarding prevalence in either sex. There was no correlation between prevalence of various infections and glycemic levels. Few patients in this study had adverse outcome which included permanent disability and mortality. HbAlc was significantly related to duration of stay and mortality.

\section{References}

[1]. Dooley KE, Chaisson RE. Tuberculosis and diabetes mellitus: Convergence of two epidemics. Lancet Infectious Diseases 2009 Dec;9(12):737-46.

[2]. Shah BR, Hux JE. Quantifying the risk of infectious diseases for people with diabetes. American Diabetes Association Diabetes Care 2003; 26(2): 510-3.

[3]. Sanjay K. Bhadada. Acute infections in diabetes mellitus. In:ChandaliaHemraj B, Sridhar Gumpeny Ramachandra, Das Ashok Kumar, Madhu Sri Venkata, Mohan Viswanathan, Rao PaturiVishnupriya, editors. RSSDI Textbook of Diabetes mellitus. $3^{\text {rd }}$ ed.NewDelhi:Jaypee Brothers Medical Publishers;2014;2:819-28.

[4]. Rassias AJ, Marrin CA, Arruda J, et al. Insulin infusion improves neutrophil function in diabetic cardiac surgery patients. Anesthesia and Analgesia 1999;88(5):1011-6.

[5]. Delamaire M, Maugendre D, Moreno M, et al. Impaired leucocyte function in diabetic patients. Diabetic Medicine 1997;14(1):2934.

[6]. The Diabetes Control and Complications Trial Research Group. The effect of intensive treatment of diabetes on the development and progression of long-term complications in insulin-dependent diabetes mellitus. NEJM 1993; 329: 977-86.

[7]. Calvet HM, Yoshikawa TT. Infections in diabetes. Infectious Diseases in Clinics of North America 2001;15(2):407-21

[8]. Wheat LJ. Infection and diabetes mellitus. American Diabetes Association Diabetes care 1980;1(3):187-97.

[9]. Rayfield EJ, Ault MJ, Keusch GT, Brothers MJ, NechemiasC,Smith H. Infection and diabetes: the case for glucose control. The American Journal of Medicine. 1982;72(3):439-50.

[10]. Mazade MA, Edwards MS. Impairment of type III group B Streptococcus-stimulated superoxide production and opsonophagocytosis by neutrophils in diabetes. Molecular Genetics and Metabolism. 2001;73(3): 259-67.(1)

[11]. Suzuki K, Kawamura T, Sakakibara F, Sasaki H, Sano T, Sakamoto N, et al. Effect of aldose reductase inhibitors on glucoseinduced changes in sorbitol and myo-inositol metabolism in human neutrophils. Diabetic medicine: A Journal of the British Diabetic Association. 1999 Jan;16(1):67-73.

[12]. Kawamura T, Suzuki K, Matsumae H, Sano T, Sakamoto N, Hotta N. Effects of glucose and SNK-860, an aldose reductase inhibitor, on the polyol pathway and chemiluminescence response of human neutrophils in vitro. Diabetic Medicine 1995;12(5): 392-6.

[13]. Anton Y.Peleg, ThilakWeerarathna, James S. McCarthy et al. Common infections in Diabetes: pathogenesis, management and relationship to glycaemic control. Diabetes Metabolism Research and Reviews 2007;23(1):3-13.

[14]. Shariq R. Masoodi, Arshad I. Wani, Raiz A. Misgar, Vipan K. Gupta, Mir I.Bashir, Abdul H. Zargar. Pattern of infections in patients with diabetesmellitus- Data from a teritary care centre in Indian sub-continent. Diabetesand metabolic syndrome: Clinical research and reviews 2007;1(2):91-5.

[15]. Nakanishi S, Yamada M, Hattori N, Suzuki G. Relationship between HbA(1)c and mortality in a Japanese population. Diabetologia. $2005 \mathrm{Feb} ; 48(2): 230-4$.

[16]. Halkos ME, Puskas JD, Lattouf OM, Kilgo P, Kerendi F, Song HK, et al.Elevated preoperative hemoglobin A1c level is predictive of adverseevents after coronary artery bypass surgery. The Journal of thoracic andcardiovascular surgery. 2008 Sep;136(3):631-40.

[17]. Gornik I, Gornik $\mathrm{O}$, Gašparović V. HbA1c is outcome predictor in diabeticpatients with sepsis. Diabetes research and clinical practice. 2007Jul;77(1),120 - 5. 